\title{
Psychological intervention to prevent ICU-related PTSD: who, when and for how long?
}

\author{
Rob Hatch', Stuart McKechnie ${ }^{1,2}$ and John Griffiths ${ }^{1,2 *}$ \\ See related research by Peris et al., http://ccforum.com/content/15/1/R41
}

\begin{abstract}
Experiencing treatment on a modern intensive care unit (ICU) is a potentially traumatic event. People who experience traumatic events have an increased risk of depression, anxiety disorders and post-traumatic stress disorder (PTSD). Extended follow-up has confirmed that many patients suffer physical and psychological consequences of the ICU treatment up to 12 months after hospital discharge. PTSD in particular has become increasingly relevant in both the immediate and longer-term follow-up care of these patients. The extent to which the consequences of critical illness and the treatments received in the ICU contribute to the development of PTSD is poorly understood and more rigorous studies are needed. Understanding the factors associated with a poor psychological recovery after critical illness is essential to generate models of causality and prognosis, and to guide the delivery of effective, timely interventions.
\end{abstract}

In the previous issue of Critical Care, Peris and coworkers demonstrate that trauma patients experience fewer future symptoms of post-traumatic stress disorder (PTSD) and a (nonsignificant) reduction in the prevalence of anxiety and depression if they receive psychological intervention whilst in the intensive care unit (ICU) [1]. These are important findings given the large number of patients who suffer significant long-term psychological disturbance during (and following) their recovery from critical illness $[2,3]$.

It is estimated that 5 to $64 \%$ of patients develop PTSD or its associated symptoms during their recovery from critical illness [4]. Studies reporting prevalence rates of PTSD in ICU survivors vary considerably in their design,

*Correspondence: john.griffiths@nda.ox.ac.uk

'Adult Intensive Care Unit, John Radcliffe Hospital, Oxford OX3 9DU, UK Full list of author information is available at the end of the article case mix and the method and timing of PTSD assessment. Thus, the extent to which the events preceding the ICU admission, the critical illness itself or the treatment received in the ICU contribute to the development of ICU-related PTSD remains poorly understood.

Survivors of nonfatal trauma appear to be at particular risk of PTSD [5], with this risk increasing significantly in those admitted to the ICU [6]. Whilst life-threatening injuries requiring ICU admission are frequently perceived as more psychologically traumatic, there is an increasing body of evidence suggesting a dependent relationship between ICU admission and the later development of PTSD, irrespective of the events preceding ICU admission. Why such a causal relationship exists is unclear. Sedation, particularly the use of uninterrupted sedative infusions [7], sleep disturbance [8,9], delirium [10], memory of the ICU experience and delusions [11] have all been implicated in the development of PTSD, as have changes in regulation of the adrenal axis $[12,13]$. ICUrelated PTSD probably results from a complex interaction between some or all of these factors. Certainly, data relating to these risk factors will be vital for the meaningful interpretation of future interventional studies of ICU-related PTSD.

The study population investigated by Peris and coworkers was clearly likely to benefit from psychological intervention as the participants were young, severely injured and were treated in the ICU for a long time. Whether specialist psychological support should be available for all critically ill patients or targeted to such at-risk groups remains unresolved. Potential risk factors that might be used to identify those at risk of post-ICU PTSD include female gender, delirium, delusional memory, traumatic memory, sedation strategy, preexisting psychiatric history and longer lengths of stay in the ICU. The development of validated screening tools to detect psychological disturbance within the ICU would also be a significant advance in facilitating targeted psychological intervention. However, given the inherent difficulties of accurately assessing the psychological state of critically ill patients, these screening tools currently remain elusive. Future development of such tools should 
involve input from our psychological and psychiatric colleagues to ensure the instruments are valid, reliable and psychometrically robust.

The optimal timing of interventions to reduce the prevalence of psychological symptoms in those recovering from critical illness remains unclear. Studies targeting ICU survivors at 6 weeks post ICU discharge [14] or at outpatient follow-up clinics [15] have shown little impact in lessening PTSD symptoms. The findings of Peris and coworkers suggest that earlier intervention may be more beneficial, with the PTSD prevalence significantly lower in patients receiving clinical psychologist support within the ICU after recovery of consciousness. The study investigators are to be congratulated for sowing the seed that early psychological intervention should be the standard of care for ICU patients and that clinical psychologists should be an integral part of a modern-day critical care multidisciplinary team to facilitate this.

The precise nature of the intervention(s) to reduce the prevalence of ICU-related PTSD has yet to be defined. Cognitive behavioural therapy has been shown to be effective in preventing PTSD in the general population [16], and the UK's National Institute of Clinical Excellence recommends that trauma-focused cognitive behavioural therapy should be offered to those with severe posttraumatic symptoms in the first month after the traumatic event. It is not clear whether patients in the study by Peris and coworkers received trauma-focused cognitive behavioural therapy. For patients treated in the ICU, simple changes in nursing practice might be all that is required to improve psychological outcomes. A recent study from the Netherlands supports this hypothesis by suggesting that the most valued aspect of nursing care is the ability to relieve fear and worry through caring behaviour [8]. Patient diaries represent another simple intervention that may lessen post-ICU psychopathology [17], and the results of RACHEL II, a multicentre study examining the effects of patient diaries on the development of PTSD, are eagerly awaited.

Nonpsychological interventions are also likely to be of importance. Sleep disturbance, sedation strategy and delirium are modifiable variables implicated in the development of PTSD. Strategies to restore physiological sleep patterns, reduce delirium and optimise analgesia and sedation should be routine in ICU practice, and are likely to be psychologically beneficial and to reduce the need for more specialised and complex psychological intervention. The role (if any) of pharmacological interventions in the treatment of PTSD within the ICU, either as an adjuvant to psychological intervention or in those unable to engage with treatments such as cognitive behavioural therapy, has yet to be defined.

Improving psychological outcomes following ICU admission is a challenge facing all who care for critically ill patients. A more detailed understanding of the factors linking critical illness with poor psychological recovery is essential to generate models of causality and to guide the delivery of targeted interventions to improve psychological outcomes. Determining the nature of effective psychological interventions in the critically ill remains a research priority. Moreover, the approach to the overall psychological care of these patients should surely be proactive (rather than reactive) and should begin during ICU admission. Demonstrating the residual symptoms and signs of psychological disturbance in ICU survivors once they have been discharged from hospital may reflect a missed opportunity of earlier, and potentially more effective, intervention. The integration of effective screening tools to identify those at risk of psychological disturbance and the establishment of early multidisciplinary psychological management into routine ICU care would be a major advance. The observational study by Peris and coworkers suggests this aspiration is achievable and is an important start.

\section{Abbreviations}

ICU, intensive care unit; PTSD, post-traumatic stress disorder.

\section{Competing interests}

The authors declare that they have no competing interests.

\section{Author details}

'Adult Intensive Care Unit, John Radcliffe Hospital, Oxford OX3 9DU, UK. 2Nuffield Department of Anaesthetics, John Radcliffe Hospital, Oxford OX3 9DU, UK.

\section{Published: 25 March 2011}

\section{References}

1. Peris A, Bonizzoli M, lozzelli D, Migliaccio ML, Zagli G, Bacchereti A, Debolini M, Vannini E, Solaro M, Balzi I, Bendoni E, Bacchi I, Giovannini V, Belloni L: Early intra-intensive care unit psychological intervention promotes recovery from post traumatic stress disorders, anxiety and depression symptoms in critically ill patients. Crit Care 2011, 15:R41.

2. Broomhead LR, Brett SJ: Clinical review: Intensive care follow-up - what has it told us? Crit Care 2002, 6:411-417.

3. Cuthbertson BH, Roughton S, Jenkinson D, Maclennan G, Vale L: Quality of life in the five years after intensive care: a cohort study. Crit Care 2010, 14:R6.

4. Griffiths J, Fortune G, Barber V, Young J: The prevalence of post traumatic stress disorder in survivors of ICU treatment: a systematic review. Intensive Care Med 2007, 33:1506-1518.

5. O'Donnell ML, Creamer M, Pattison P, Atkin C: Psychiatric morbidity following injury. Am J Psychiatry 2004, 161:507-514.

6. O'Donnell ML, Creamer M, Holmes AC, Ellen S, MCFarlane AC, Judson R, Silove D, Bryant RA: Posttraumatic stress disorder after injury: does admission to intensive care unit increase risk? J Trauma 2010, 69:627-632

7. Kress JP, Gehlbach B, Lacy M, Pliskin N, Pohlman AS, Hall JB: The long-term psychological effects of daily sedative interruption on critically ill patients. Am J Respir Crit Care 2003, 168:1457-1461.

8. Hofhuis J, Spronk P, Van Stel H, Schrijvers A, Rommes J, Bakker J: Experiences of critically ill patients in the ICU. Intensive Crit Care Nurs 2008, 24:300-313.

9. Mellman TA, Hipolito MM: Sleep disturbances in the aftermath of trauma and posttraumatic stress disorder. CNS Spectr 2006, 11:611-615.

10. Salluh JI, Soares M, Teles JM, Ceraso D, Raimondi N, Nava VS, Blasquez P, Ugarte S, Ibanez-Guzman C, Centeno JV, Laca M, Grecco G, Jimenez E, AriasRivera S, Duenas C, Rocha MG: The DECCA (Delirium Epidemiology in Critical Care) Study Group. Delirium epidemiology in critical care (DECCA): an international study. Crit Care 2010, 14:R210. 
11. Jones C, Griffiths RD, Humphris G, Skirrow PM: Memory, delusions, and the development of acute posttraumatic stress disorder-related symptoms after intensive care. Crit Care Med 2003, 29:573-580.

12. Hauer D, Weis F, Krauseneck T, Vogeser M, Schelling G, Roozendaal B: Traumatic memories; post-traumatic stress disorder and serum cortisol levels in long-term survivors of the acute respiratory distress syndrome. Brain Res 2009, 1293:114-120.

13. Weis F, Kilger E, Roozendaal B, de Quervain DJ, Lamm P, Schmidt M, Schmolz $M$, Briegel J, Schelling G: Stress doses of hydrocortisone reduce chronic stress symptoms and improve health-related quality of life in high-risk patients after cardiac surgery: a randomized study. J Thorac Cardiovasc Surg 2006, 131:277-282

14. Jones C, Skirrow P, Griffiths RD, Humphris GH, Ingleby S, Eddleston J, Waldmann C, Gager M: Rehabilitation after critical illness: a randomized, controlled trial. Crit Care Med 2003, 31:2456-2461.

15. Cuthbertson BH, Rattray J, Campbell MK, Roughton S, Smith A, Hull A, Breeman S, Norrie J, Jenkinson D, Hernández R, Johnston M, Wilson E,
Waldmann C: The PRaCTICaL study of nurse led, intensive care follow-up programmes for improving long term outcomes from critical illness: a pragmatic randomised controlled trial. BMJ 2009, 339:b3723.

16. Bryant RA, Mastrodomenico J, Felmingham KL, Hopwood S, Kenny L, Kandris E, Cahill C, Creamer M: Treatment of acute stress disorder: a randomized controlled trial. Arch Gen Psychiatry 2008, 65:659-667.

17. Knowles R, Tarrier N: Evaluation of the effect of prospective patient diaries on emotional well-being in intensive care unit survivors: a randomized controlled trial. Crit Care Med 2009, 3:184-191.

doi:10.1186/cc10054

Cite this article as: Hatch $\mathrm{R}$, et al:: Psychological intervention to prevent ICU-related PTSD: who, when and for how long? Critical Care 2011, 15:141. 